\author{
A. Banerjee, S. Bhattacharyya
}

\title{
MEROMORPHIC FUNCTION SHARING THREE SETS WITH ITS SHIFT, $q$-SHIFT, $q$-DIFFERENCE AND ANALOGOUS OPERATORS
}

\begin{abstract}
A. Banerjee, S. Bhattacharyya. Meromorphic function sharing three sets with its shift, q-shift, q-difference and analogous operators, Mat. Stud. 52 (2019), 38-47.

Using the notion of weighted sharing of three sets we deal with the uniqueness problem of meromorphic function with its shift, $q$-shift, $q$-difference and analogous operator. In other words, we deduce conditions providing a) $f(z) \equiv t f(z+c)$, where $t^{n}=1, n \geq 2$; b) $f(z) \equiv t f(q z+c)$, where $t^{n}=1, n \geq 2$ and $q, c \in \mathbb{C} \backslash\{0\} ;$ c) $f(z) \equiv t f(q z)$, where $t^{n}=1$ and $\left.|q|=1 ; \mathrm{d}\right)$ $f(z) \equiv \chi_{n} \Delta_{q} f$ for $n \geq 2$ with $\Delta_{q} f=f(q z)-f(z)$ and $\chi_{n}=\left\{\begin{array}{ll}1, & \text { if } n=2, \\ t, & \text { if } n \geq 3, t^{n}=1 .\end{array}\right.$ A handful
\end{abstract} number of examples have been provided to substantiate our certain claims.

1. Introduction definitions and results. Throughout the paper, the term "meromorphic" will be used to mean meromorphic in the whole complex plane. Let $f$ and $g$ be such two non-constant meromorphic functions defined in the open complex plane $\mathbb{C}$. For some $a \in \mathbb{C}$, we denote by $E(a ; f)$ the set of the zeros of $f-a$, where every zero is counted according to its multiplicity. In addition to this, when $a=\infty$, the above definition implies that we are considering the poles. In the same manner, by $\bar{E}(a ; f)$, we denote the collection of the distinct zeros or poles of $f-a$ according as $a \in \mathbb{C}$ or $a=\infty$ respectively. If $E(a ; f)=E(a ; g)$ we say that $f$ and $g$ share the value $a \mathrm{CM}$ (counting multiplicities) and if $\bar{E}(a ; f)=\bar{E}(a ; g)$, then we say that $f$ and $g$ share the value $a$ IM (ignoring multiplicities).

Let $S$ be a set of distinct elements of $\mathbb{C} \cup\{\infty\}$ and $E_{f}(S)=\bigcup_{a \in S}\{z: f(z)-a=0\}$, where each zero is counted according to its multiplicity. If we do not count the multiplicity then the set $\bigcup_{a \in S}\{z: f(z)-a=0\}$ is denoted by $\bar{E}_{f}(S)$.

If $E_{f}(S)=E_{g}(S)$ we say that $f$ and $g$ share the set $S$ CM. On the other hand, if $\bar{E}_{f}(S)=\bar{E}_{g}(S)$, we say that $f$ and $g$ share the set $S$ IM.

Usually, $S(r, f)$ denotes any quantity satisfying $S(r, f)=o(T(r, f))$ for all $r$ outside of a possible exceptional set of finite linear measure. Also $S_{1}(r, f)$ denotes any quantity satisfying $S_{1}(r, f)=o(T(r, f))$ for all $r$ on a set of logarithmic density 1, where the logarithmic density of a set $F$ is defined by

$$
\limsup _{r \rightarrow \infty} \frac{1}{\log r} \int_{[1, r] \cap F} \frac{d t}{t} .
$$

2010 Mathematics Subject Classification: 30D35.

Keywords: meromorphic functions; uniqueness; shift; difference operator; shared set. doi:10.30970/ms.52.1.38-47 
For $q \in \mathbb{C} \backslash\{0\}$ we define shift and $q$-shift operators of a non-constant meromorphic function by $f(z+c)$ and $f(q z)$, respectively.

Also, for $q \in \mathbb{C} \backslash\{0,1\}$ we define $q_{c}$-shift and $q$-difference operators of a non-constant meromorphic function by $f(q z+c)$ and $\Delta_{q} f=f(q z)-f(z)$ respectively.

In this paper $S_{1}, S_{2}$ and $S_{3}$ represents the sets $\left\{1, \omega, \omega^{2}, \ldots, \omega^{n-1}\right\},\{0\}$ and $\{\infty\}$ respectively, where $\omega=\cos \frac{2 \pi}{n}+i \sin \frac{2 \pi}{n}$ and $n$ is a positive integer.

We denote

$$
\chi_{n}= \begin{cases}1, & \text { if } n=2, \\ t, & \text { if } n \geq 3, t^{n}=1\end{cases}
$$

In 1982, concerning two shared set problems, Gross-Osgood [4] employed the order notion on entire function to find the relation between them.

Theorem A. Let $S_{1}=\{1,-1\}, S_{2}=\{0\}$. If $f$ and $g$ are non-constant entire functions of finite order such that $E_{f}\left(S_{j}, \infty\right)=E_{g}\left(S_{j}, \infty\right)$ for $j=1,2$, then $f= \pm g$ or $f g=1$.

So it is interesting to investigate Theorem $\mathrm{A}$ in the case of two non-entire meromorphic functions. Let $f(z)=\frac{e^{z}}{A+B e^{z}}$ and $g(z)=\frac{1}{A e^{z}+B}$ with $A^{2}=B^{2}-1 ; A, B \in \mathbb{C} \backslash\{0\}$. It is easy to verify that the functions satisfy the sharing conditions of Theorem A but the conclusion does not hold.

So one can presume that, for non-entire meromorphic functions, in order to obtain the conclusion of Theorem A, pole sharing is essential.

However, in course of time, researchers were ultimately succeeded to remove the order restrictions in the shared set problems and consequently many important and elegant results were obtained.

In the meantime in 2001, the introduction of the notion of weighted sharing [8,9], of values and sets, which is actually a scaling between CM and IM sharing, further add essence to the literature. Below we invoke the definition.

Definition 1 ([8,9]). Let $k$ be a non-negative integer or infinity. For $a \in \mathbb{C} \cup\{\infty\}$ we denote by $E_{k}(a ; f)$ the set of all $a$-points of $f$, where an a-point of multiplicity $m$ is counted $m$ times if $m \leq k$ and $k+1$ times if $m>k$. If $E_{k}(a ; f)=E_{k}(a ; g)$, we say that $f, g$ share the value $a$ with weight $k$ and denote it by $(a, k)$. The IM and CM sharing corresponds to $(a, 0)$ and $(a, \infty)$, respectively.

Definition 2 ([8]). Let $S$ be a set of distinct elements of $\mathbb{C} \cup\{\infty\}$ and $k$ be a non-negative integer or $\infty$. We denote by $E_{f}(S, k)$ the set $\bigcup_{a \in S} E_{k}(a ; f)$. Clearly $E_{f}(S)=E_{f}(S, \infty)$ and $\bar{E}_{f}(S)=E_{f}(S, 0)$.

In 2006, Halburd-Korhonen [5] obtained difference analogue of the logarithmic derivative lemma for finite order meromorphic function. In the next year, the same analogous result corresponding to $f(q z)$ for zero-order meromorphic function was discovered in [2].

These two results induced great interest among the researchers to again ponder over the effect of order notion in shared set problem in the uniqueness theory. Naturally researchers became engaged to investigate the problems of meromorphic functions sharing sets with its respective shift, difference, $q_{c}$-shift $q$-difference operators.

In 2010, considering the relation between $f(z)$ and its shift $f(z+c)$ sharing two sets, Zhang [16] obtained the following result. 
Theorem B $([16])$. Let $c \in \mathbb{C}$. Suppose $f(z)$ is a non-constant meromorphic function of finite order such that $E_{f(z)}\left(S_{j}, \infty\right)=E_{f(z+c)}\left(S_{j}, \infty\right) j=1,3$ for $n \geq 4$, then $f(z) \equiv t f(z+c)$, where $t^{n}=1$.

In 2013, concerning $f$ and $\Delta_{q} f$, sharing the sets $S_{1}$ and $S_{3}$, Qi-Yang [10] obtained the following theorem.

Theorem C ([10]). Considering the set $S_{1}$ and $S_{3}$, let $f$ is a non-constant zero-order meromorphic function such that $E_{f}\left(S_{j}, \infty\right)=E_{\Delta_{q} f}\left(S_{j}, \infty\right)$, for $j=1,3$ and $q \in \mathbb{C} \backslash\{0\}$. If $n \geq 5$, then $f(z)=t \Delta_{q} f$, where $t^{n}=1$.

In the next year, Qi-Yang [11] investigated the relation between $f(z)$ and $f(q z)$ under two shared sets problems. They [11] proved the result as shown below.

Theorem $\mathbf{D}([11])$. Considering the sets $S_{1}$ and $S_{3}$, if $f$ is a non-constant zero-order meromorphic function such that $E_{f}\left(S_{j}, \infty\right)=E_{f(q z)}\left(S_{j}, \infty\right)$ for $j=1,3$, and $q \in \mathbb{C} \backslash\{0\}$. If $n \geq 4$, then $f(z) \equiv t f(q z), t^{n}=1$ and $|q|=1$.

So it is natural to ask the following question of the paper.

1. Is it possible to reduce further the lower bound of $n$ in Theorem B, Theorem $\mathrm{C}$ and Theorem D?

In the paper we have been able to reduce the lower bound at the cost of considering an additional set namely the set $S_{2}$ by using the notion of weighted sharing.

For the sake of convenience let us denote the expression $2 m p k+(p+k)(m-1)$ by $\psi_{p, k}^{m}$.

The following theorems are the main results of the paper.

Theorem 1. Let $c \in \mathbb{C}$. Suppose $f(z)$ be a non-constant meromorphic function of finite order such that $E_{f(z)}\left(S_{1}, m\right)=E_{f(z+c)}\left(S_{1}, m\right), E_{f(z)}\left(S_{2}, p\right)=E_{f(z+c)}\left(S_{2}, p\right)$ and $E_{f(z)}\left(S_{3}, k\right)=$ $E_{f(z+c)}\left(S_{3}, k\right)$. If $n \geq 2$ and $\psi_{p, k}^{m}>2$ then $f(z)=t f(z+c)$, where $t^{n}=1$.

The following example shows that in Theorem 1 for $n=2$, sharing of the set $S_{1}$ is essential.

Example 1. Let

$$
f(z)=\frac{\phi\left(\frac{\pi z}{2 c}\right)}{A+B \sin ^{2 s}\left(\frac{\pi z}{2 c}\right) \cos ^{2 s}\left(\frac{\pi z}{2 c}\right)},
$$

where $s$ is a positive integer and $A, B \in \mathbb{C} \backslash\{0\}$. Then for two suitable choice of $\phi(z)$ as $\sin ^{2 s} z \cos ^{2 s+2} z$ or $\sin ^{2 s+1} z \cos ^{2 s-1} z, f(z)$ and $f(z+c)$ share $\left(S_{2}, 2 s-1\right)$ or $\left(S_{2}, 2 s-2\right)$, respectively, and they share $\left(S_{3}, \infty\right)$. But $f(z) \neq t f(z+c)$, for any real $t$.

Like the above example in the next two examples we exhibit that in Theorem 1 for $n=2$, sharing of the set $S_{2}$ is essential.

Example 2. For an integer $r$, let $f(z)=\sqrt{2} \sin \frac{(2 r+1) \pi z}{2 c}$ or $\sqrt{2} \cos \frac{(2 r-1) \pi z}{2 c}$. Then clearly $E_{f(z)}\left(S_{j}, \infty\right)=E_{f(z+c)}\left(S_{j}, \infty\right)$, for $j=1$, 3. But $f(z) \neq t f(z+c)$, for any real $t$.

Example 3. Let

$$
f(z)=\frac{\exp \left(\frac{\pi i z}{2 c}\right)-\exp \left(-\frac{\pi i z}{2 c}\right) A^{2}}{\sqrt{2} i A},
$$

where $A \in \mathbb{C} \backslash\{0\}$. Clearly $E_{f(z)}\left(S_{j}, \infty\right)=E_{f(z+c)}\left(S_{j}, \infty\right)$, for $j=1$, 3. But $f(z) \neq t f(z+c)$, for any real $t$. 
The following example shows that the conclusion of Theorem 1 actually occurs for $n=2$.

Example 4. Let

$$
f(z)=\frac{\sin \left(\frac{\pi z}{c}\right)}{1+\sin ^{2}\left(\frac{\pi z}{c}\right)} .
$$

Then $f(z+c)=-f(z)$ share $S_{i}$, for $i=1,2,3$ and clearly we see that $f(z)=t f(z+c)$, where $t^{n}=1$ holds.

The following example shows that Theorem 1 is not valid for a function of infinite order for $n=2$.

Example 5. Let $f(z)=e^{e^{z}}$. Choosing $e^{c}=-1$, it is easy to verify that $f(z)$ and $f(z+c)$ share $\left(S_{i}, \infty\right)$ for $i=1,2,3$; but $f(z) \neq t f(z+c)$, where $t^{n}=1$.

Theorem 2. Let $f(z)$ be a non-constant zero-order meromorphic function such that $E_{f(z)}\left(S_{1}, m\right)=E_{f(q z+c)}\left(S_{1}, m\right), E_{f(z)}\left(S_{2}, p\right)=E_{f(q z+c)}\left(S_{2}, p\right), E_{f(z)}\left(S_{3}, k\right)=E_{f(q z+c)}\left(S_{3}, k\right)$ and $q, c \in \mathbb{C} \backslash\{0\}$. If $n \geq 2$ and $\psi_{p, k}^{m}>2$ then $f(z)=t f(q z+c)$, where $t^{n}=1$.

In the following example we see that the conclusion of Theorem 2 actually holds for $n=2$.

Example 6. Let $f(z)=\frac{z(z-1)(2 z-1)}{1+z(z-1)}$. Then clearly for $q=-1$ and $c=1$, we have $E_{f(z)}\left(S_{i}, \infty\right)=E_{f(q z+c)}\left(S_{i}, \infty\right)$; for $i=1,2,3$ and $f(q z+c)=-f(z)$.

The next example exhibits that in Theorem 2 for $n=2$ sharing of the set $S_{1}$ is essential.

Example 7. Let $f(z)=\frac{z^{2 r}(z-1)^{2 r-1}}{1+z^{2 r-1}(z-1)^{2 r-1}}$. Then clearly for $q=-1$ and $c=1$, we have $E_{f(z)}\left(S_{i}, \infty\right)=E_{f(q z+c)}\left(S_{i}, \infty\right)$; for $i=1,3$ and $E_{f(z)}\left(S_{2}, 2 r-2\right)=E_{f(q z+c)}\left(S_{2}, 2 r-2\right)$. But $f(z) \neq t f(q z+c)$, for any real $t$.

The following two examples show that under the assumptions of Theorem 2 with $n=2$, for non-zero finite order meromorphic function $f$, the same conclusion may or may not hold respectively.

Example 8. Let $f(z)=\frac{\sin \left(\frac{2 \pi z}{c}\right)}{1+\sin ^{2}\left(\frac{\pi z}{c}\right)}$ and for $q=-1, f(q z+c)=-\frac{\sin \left(\frac{2 \pi z}{c}\right)}{1+\sin ^{2}\left(\frac{\pi z}{c}\right)}$. Then clearly $E_{f(z)}\left(S_{i}, \infty\right)=E_{f(q z+c)}\left(S_{i}, \infty\right)$ holds, for $i=1,2,3$ and also we see that $f(z)=-f(q z+c)$.

Example 9. Let $f(z)=A e^{z}+B$ and so $f(q z+c)=A D e^{-z}+B$ with $A^{2} D=B^{2}-1$, where $A, B, D \in \mathbb{C} \backslash\{0\}$ and $q=-1$ and $e^{c}=D$. Then $E_{f(z)}\left(S_{i}, \infty\right)=E_{f(q z+c)}\left(S_{i}, \infty\right)$ holds, for $i=1,2,3$, but $f(z) \neq t f(q z+c)$, where $t^{2}=1$.

Theorem 3. Let $f(z)$ be a non-constant zero-order meromorphic function such that

$$
E_{f(z)}\left(S_{1}, m\right)=E_{f(q z)}\left(S_{1}, m\right), E_{f(z)}\left(S_{2}, p\right)=E_{f(q z)}\left(S_{2}, p\right), E_{f(z)}\left(S_{3}, k\right)=E_{f(q z)}\left(S_{3}, k\right)
$$

and $q \in \mathbb{C} \backslash\{0\}$. If $n \geq 2$ and $\psi_{p, k}^{m}>2$ then $f(z)=t f(q z)$, where $t^{n}=1$ and $|q|=1$.

The following two examples show that the conclusion of Theorem 3 actually occurs for the case $n=2$ and $n=3$, respectively. 
Example 10. Let $f(z)=\left(A z^{2 m+1}+B z^{2 m+3}\right)^{-1}$. Then for $q=-1, f(q z)=-\left(A z^{2 m+1}+\right.$ $\left.B z^{2 m+3}\right)^{-1}$, where $m$ is an integer. Then it can easily be verified that $E_{f(z)}\left(S_{i}, \infty\right)=$ $E_{f(q z)}\left(S_{i}, \infty\right)$, for $i=1,2,3$ and $f(z)=-f(q z)$.

Example 11. Let $f(z)=\frac{z^{2 r}}{1+z^{6 r}}$, where $r$ is an integer. Then for $q= \pm \omega, \pm \omega^{2}$, it is easy to verify that $f(z)$ and $f(q z)$ share $\left\{1, \omega, \omega^{2}\right\},\{0\},\{\infty\}$ and $f(z)=t f(q z)$, where $t^{3}=1$.

The following example shows that if $f$ is of non-zero finite order with $\sigma(f) \geq 1, f(z)$ and $f(q z)$ share the sets $S_{i}, i=1,2,3$, then for $n=2$, the conclusion of Theorem 3 may or may not hold. Unfortunately we have not been able to produce any counterexample for the situation when any $f$ with $0<\sigma(f)<1$ satisfies the conditions of Theorem 3 .

Example 12. Let $f(z)=\frac{\sin z}{1+\sin ^{2} z}$. Then $f(q z)=-\frac{\sin z}{1+\sin ^{2} z}$ for $q=-1$. Thus, clearly $E_{f(z)}\left(S_{i}, \infty\right)=E_{f(q z)}\left(S_{i}, \infty\right)$ holds, for $i=1,2,3$ and also we see that $f(z)=-f(q z)$.

Example 13. Let $f(z)=A e^{z}+B$ and so $f(q z)=A e^{-z}+B$ with $A^{2}=B^{2}-1$, where $A, B \in \mathbb{C} \backslash\{0\}$ and $q=-1$. Then $E_{f(z)}\left(S_{i}, \infty\right)=E_{f(q z)}\left(S_{i}, \infty\right)$ holds for $i=1,2,3$, but $f(z) \neq t f(q z)$, where $t^{2}=1$.

Theorem 4. Let $f(z)$ be a non-constant zero-order meromorphic function such that $E_{f(z)}\left(S_{1}, m\right)=E_{\Delta_{q} f}\left(S_{1}, m\right), E_{f(z)}\left(S_{2}, p\right)=E_{\Delta_{q} f}\left(S_{2}, p\right)$ and $E_{f(z)}\left(S_{3}, k\right)=E_{\Delta_{q} f}\left(S_{3}, k\right)$ and $q \in \mathbb{C} \backslash\{0\}$. If $n \geq 2$ and $\psi_{p, k}^{m}>2$ then $f(z)=\chi_{n} \Delta_{q} f$.

Remark 1. For Theorem 4 if we choose $f(q z)=\left(1+\tau^{n-1}\right) f(z)$, where $n \geq 3$ and $\tau$ is any one of the generators of the group of $n$-th root of unity, then $\Delta_{q} f$ and $f(z)$ satisfy the hypothesis of Theorem 4 . For $n=2$ we need to choose $f(q z)=2 f(z)$.

The following two examples establish the fact that Theorem 4 holds true in the case of $n=3$ for entire and meromorphic functions respectively.

Example 14. Let $f(z)=z$. Then for $q=-\omega$ we get $\Delta_{q} f=-(\omega+1) z$. Clearly we see that $f(z)$ and $\Delta_{q} f$ share the sets $S_{1}, S_{2}, S_{3}$ and also the condition $f(z)=\chi_{n} \Delta_{q} f$ holds.

Example 15. Let $f(z)=\frac{z^{6 r+1}}{1+z^{6 r}}$. Then for $q=-w$ we get $\Delta_{q} f=-(1+w) \frac{z^{6 r+1}}{1+z^{6 r}}$, where $r$ is integer. Clearly it can be seen that $f(z)$ and $\Delta_{q} f$ share the sets $S_{1}, S_{2}, S_{3}$ and the condition $f(z)=\chi_{n} \Delta_{q} f$ holds.

Again the next two examples show that Theorem 4 holds for $n=4$ for both entire and meromorphic functions.

Example 16. Let $f(z)=z$ and then for $q=(1+i)$ or $1-i$ it is easy to verify that that $f(z)$ and $\Delta_{q} f$ share the sets $S_{1}, S_{2}, S_{3}$ and also the condition $f(z)=\chi_{n} \Delta_{q} f$ holds.

Example 17. Let $f(z)=\frac{1}{z^{n}}$. Then for $q^{n}=\frac{1-i}{2}$ or $\frac{1+i}{2}$, we see that $f(z)$ and $\Delta_{q} f$ share the sets $S_{1}, S_{2}, S_{3}$ and the condition $f(z)=\chi_{n} \Delta_{q} f$ holds.

Now in the following example we show that the set $S_{1}$ is essential for any $n(\geq 2)$.

Example 18. Let $f(z)=\frac{z^{2 r-1}}{1+z^{4 r}}$, where $r$ is a positive integer. Then for $q=i$ it is easy to verify that $f(z)$ and $\Delta_{q} f$ share the sets $S_{2}$ and $S_{3}$ but do not share the set $S_{1}$, in Theorem 4 , for any $n \geq 2$. Also the condition $f(z)=\chi_{n} \Delta_{q} f$ is not satisfied. 
Corollary 1. Theorems 1, 2, 3 and 4 hold for the following minimal triplets of values of $m$, $p$ and $k$ :

(i) $m=1, p=1, k=2 ; \quad$ (ii) $m=1, p=2, k=1 ; \quad$ (iii) $m=2, p=0, k=3$;

(iv) $m=2, p=1, k=1$; (v) $m=2, p=2, k=1$; (vi) $m=3, p=0, k=2$;

(vii) $m=3, p=1, k=1 ; \quad$ (viii) $m=3, p=2, k=0$.

For the standard definitions and notations of the value distribution theory we refer to [6]. But in the paper we have used some more notations and definitions which are explained below.

Definition $3([7])$. For $a \in \mathbb{C} \cup\{\infty\}$ and for a positive integer $m$ we denote by $N(r, a ; f \mid \leq$ $m)(N(r, a ; f \mid \geq m))$ the counting function of those a-points of $f$ whose multiplicities are not greater(less) than $m$ where each a-point is counted according to its multiplicity.

$\bar{N}(r, a ; f \mid \leq m)(\bar{N}(r, a ; f \mid \geq m))$ are defined similarly, where in counting of the a-points of $f$ we ignore the multiplicities.

Also, $N(r, a ; f \mid<m), N(r, a ; f \mid>m), \bar{N}(r, a ; f \mid<m)$ and $\bar{N}(r, a ; f \mid>m)$ are defined analogously.

2. Lemmas. In this section some lemmas will be presented which will be needed in the sequel. Let $F$ and $G$ be two non-constant meromorphic functions defined in $\mathbb{C}$. Henceforth we shall denote by $H, U, V$ and $W$ the following functions

$$
\begin{gathered}
U=\frac{F^{\prime}}{F-1}-\frac{G^{\prime}}{G-1}, \quad W=\frac{F^{\prime}}{F}-\frac{G^{\prime}}{G}, \\
V=\left(\frac{F^{\prime}}{F-1}-\frac{F^{\prime}}{F}\right)-\left(\frac{G^{\prime}}{G-1}-\frac{G^{\prime}}{G}\right)=\frac{F^{\prime}}{F(F-1)}-\frac{G^{\prime}}{G(G-1)}, \\
H=\left(\frac{F^{\prime \prime}}{F^{\prime}}-\frac{2 F^{\prime}}{F-1}\right)-\left(\frac{G^{\prime \prime}}{G^{\prime}}-\frac{2 G^{\prime}}{G-1}\right) .
\end{gathered}
$$

Lemma 1 ([3]). Let $f(z)$ be a meromorphic function of finite order $\rho$ and let $c \in \mathbb{C} \backslash\{0\}$ be fixed. Then, for each $\varepsilon>0$, we have

$$
T(r, f(z+c))=T(r, f(z))+O\left(r^{\rho-1+\varepsilon}\right)+O(\log r) .
$$

Lemma 2 ([5]). Let $f(z)$ be a meromorphic function of finite order and $c \in \mathbb{C} \backslash\{0\}$. Then

$$
m\left(r, \frac{f(z+c)}{f(z)}\right)+m\left(r, \frac{f(z)}{f(z+c)}\right)=S(r, f) .
$$

Lemma $3([2])$. Let $f(z)$ be a zero-order meromorphic function and let $q \in \mathbb{C} \backslash\{0\}$. Then

$$
m\left(r, \frac{f(q z)}{f(z)}\right)=S_{1}(r, f) .
$$

Lemma 4 ([17]). Let $f$ be a zero-order meromorphic function and $q \in \mathbb{C} \backslash\{0\}$. Then

$$
T(r, f(q z))=(1+o(1)) T(r, f(z)) \text { and } N(r, f(q z))=(1+o(1)) N(r, f(z))
$$

on a set of lower logarithmic density 1.

Remark 2. Thus, from the definition of $S_{1}(r, f)$ from Lemma 4 we have

$$
T(r, f(q z))=T(r, f(z))+S_{1}(r, f), \quad N(r, f(q z))=N(r, f(z))+S_{1}(r, f) .
$$


Lemma 5 ([12]). Let $f(z)$ be a non-constant zero-order meromorphic function and $q \in$ $\mathbb{C} \backslash\{0\}$, then

$$
T(r, f(q z+c))=(1+o(1)) T(r, f(z))+S_{1}(r, f)
$$

on a set of lower logarithmic measure 1.

Remark 3. Let $f(z)$ be a non-constant zero-order meromorphic function and $q \in \mathbb{C} \backslash\{0\}$, then

$$
N(r, f(q z+c))=(1+o(1)) N(r, f(z))+S_{1}(r, f)
$$

on a set of lower logarithmic measure 1.

Lemma $6([14,15])$. Let $F$ and $G$ be two non-constant meromorphic functions sharing $(1,0)$. If

$$
\bar{N}(r, 0 ; F)+\bar{N}(r, \infty ; F)=S(r, F) \text { and } \bar{N}(r, 0 ; G)+\bar{N}(r, \infty ; G)=S(r, G)
$$

then $F \equiv G$ or $F G \equiv 1$.

Let

$$
F=f^{n} \text { and } G=g^{n},
$$

where $f, g$ are non-constant meromorphic functions and $n(\geq 2)$ is an integer.

From Lemma 6 we immediately deduce the following result.

Remark 4. Suppose that $F, G$ share $(1, m),(0, p),(\infty, k)$ and $F, G$ be given by (1). If

$$
\begin{gathered}
\bar{N}(r, 0 ; F \mid \geq n p+n)+\bar{N}(r, \infty ; F \mid \geq n k+n)+\bar{N}(r, 0 ; G \mid \geq n p+n)+ \\
+\bar{N}(r, \infty ; G \mid \geq n k+n)=S(r, f)+S(r, g)
\end{gathered}
$$

then $f \equiv t g$, where $t^{n}=1$, or $f . g \equiv s$, where $0, \infty$ are lacunary values of $f$ and $g$ and $s^{n}=1$.

Proof. For $a=\{0\}$ or $\{\infty\}$, from Lemma 6 we notice that

$\bar{N}\left(r, a ; f^{n}\right)=\bar{N}\left(r, a ; f^{n} \mid=n\right)+\bar{N}\left(r, a ; f^{n} \mid=2 n\right)+\ldots+\bar{N}\left(r, a ; f^{n} \mid \geq n k+n\right)=S(r, f)$.

This implies $\bar{N}(r, a ; f \mid \geq n k+n)=S(r, f)$. Hence we have

$$
\begin{gathered}
\bar{N}(r, 0 ; F \mid \geq n p+n)+\bar{N}(r, \infty ; F \mid \geq n k+n)+\bar{N}(r, 0 ; G \mid \geq n p+n)+ \\
+\bar{N}(r, \infty ; G \mid \geq n k+n)=S(r, f)+S(r, g) .
\end{gathered}
$$

Lemma 7 ([13]). Let $f$ be a non-constant meromorphic function and $P(f)=a_{0}+a_{1} f+$ $a_{2} f^{2}+\ldots+a_{n} f^{n}$, where $a_{0}, a_{1}, a_{2} \ldots, a_{n}$ are constants and $a_{n} \neq 0$. Then $T(r, P(f))=$ $n T(r, f)+O(1)$.

Lemma 8 ([14]). Let $F, G$ be given by (1). If $F, G$ share $(0,0)$ and $U \equiv 0$ then $F \equiv G$.

Lemma 9 ([14]). Let $F, G$ be given by (1). If $F, G$ share $(\infty, 0)$ and $V \equiv 0$ then $F \equiv G$.

Lemma 10 ([1]). Let $F, G$ be given by (1). If $F, G$ share $(1,0)$ and $W \equiv 0$ then $F \equiv G$. 
Lemma 11. Let $F, G$ be given by (1) and $F \not \equiv G$. If $E_{f}\left(S_{1}, m\right)=E_{g}\left(S_{1}, m\right), E_{f}\left(S_{2}, p\right)=$ $E_{g}\left(S_{2}, p\right)$ and $E_{f}\left(S_{3}, k\right)=E_{g}\left(S_{3}, k\right)$, where $1 \leq m<\infty, 0 \leq p<\infty, 0 \leq k<\infty$ then

$$
\begin{gathered}
\left(n p+n-1-\frac{n k+n+m+1}{m(n k+n-1)-1}\right)(\bar{N}(r, 0 ; F \mid \geq n p+n)+\bar{N}(r, 0 ; G \mid \geq n p+n)) \leq \\
\leq S(r, f)+S(r, g) .
\end{gathered}
$$

Proof. Since $F \not \equiv G$ we have from Lemmas 8,9 and 10 that $U \not \equiv 0, V \not \equiv 0$ and $W \not \equiv 0$. According to the statement of the lemma it is clear that $F, G$ share $(1, m),(0, n p),(\infty ; n k)$. Also if $z_{0}$ is a zero (pole) of $f$ of order $s \leq p,(s \leq k)$ then it will be a zero (pole) of $F, G$ of order $(n-1) s+s(n s)$. Also, if $z_{0}$ is a zero (pole) of $F$ with multiplicity $r \geq n p+1(\geq n k+1)$ then it is a zero (pole) of $G$ with multiplicity $s \geq n p+1(\geq n k+1)$ and vice versa. We note that any zero (pole) of $f$ or $g$ which is also a zero (pole) of $F$ and $G$ can not lie between $(n-1) p+p$ and $(n-1) p+p+n(n k$ and $n k+n)$.

Hence, we get from the definition of $U$

$$
\begin{gathered}
(n p+n-1) \bar{N}(r, 0 ; F \mid \geq n p+n) \leq N(r, 0 ; U) \leq \\
\leq T(r, U)+O(1) \leq N(r, \infty ; U)+S(r, f)+S(r, g) \\
\leq \bar{N}(r, \infty ; F \mid \geq n k+n)+\bar{N}(r, 1 ; F \mid \geq m+1)+S(r, f)+S(r, g) .
\end{gathered}
$$

Similarly as above we get from the definition of $V$

$$
\begin{gathered}
(n k+n-1) \bar{N}(r, \infty ; F \mid \geq n k+n) \leq N(r, 0 ; V) \leq \\
\leq N(r, \infty ; V)+S(r, f)+S(r, g) \leq \\
\leq \bar{N}(r, 0 ; F \mid \geq n p+n)+\bar{N}(r, 1 ; F \mid \geq m+1)+S(r, f)+S(r, g) .
\end{gathered}
$$

Also from the definition of $W$ we deduce

$$
\begin{gathered}
m \bar{N}(r, 1 ; F \mid \geq m+1) \leq N(r, \infty ; W)+S(r, f)+S(r, g) \leq \\
\leq \bar{N}(r, 0 ; F \mid \geq n p+n)+\bar{N}(r, \infty ; F \mid \geq n k+n)+S(r, f)+S(r, g) .
\end{gathered}
$$

Using (5) in (4) we obtain

$$
\bar{N}(r, \infty ; F \mid \geq n k+n) \leq \frac{m+1}{m(n k+n-1)-1} \bar{N}(r, 0 ; F \mid \geq n p+n)+S(r, f)+S(r, g) .
$$

Using (6) in (5) we obtain

$$
\bar{N}(r, 1 ; F \mid \geq m+1) \leq \frac{n k+n}{m(n k+n-1)-1} \bar{N}(r, 0 ; F \mid \geq n p+n)+S(r, f)+S(r, g) .
$$

Now using (6) and (7) in (3) we get

$$
\left(n p+n-1-\frac{n k+n+m+1}{m(n k+n-1)-1}\right) \bar{N}(r, 0 ; F \mid \geq n p+n) \leq S(r, f)+S(r, g) .
$$

Similarly, we have

$$
\left(n p+n-1-\frac{n k+n+m+1}{m(n k+n-1)-1}\right) \bar{N}(r, 0 ; G \mid \geq n p+n) \leq S(r, f)+S(r, g) .
$$

Adding (8) and (9), we get the required conclusion of the lemma. 
Lemma 12. Let $F, G$ be given by (1) and $F \not \equiv G$. If $E_{f}\left(S_{1}, m\right)=E_{g}\left(S_{1}, m\right) ; E_{f}\left(S_{2}, p\right)=$ $E_{g}\left(S_{2}, p\right)$ and $E_{f}\left(S_{3}, k\right)=E_{g}\left(S_{3}, k\right)$, where $1 \leq m<\infty, 0 \leq p<\infty, 0 \leq k<\infty$, then

$$
\begin{gathered}
\left(n k+n-1-\frac{n p+n+m+1}{m(n p+n-1)-1}\right)(\bar{N}(r, \infty ; F \mid \geq n k+n)+\bar{N}(r, \infty ; G \mid \geq n k+n)) \leq \\
\leq S(r, f)+S(r, g) .
\end{gathered}
$$

Proof. In the line of Lemma 11, we can prove the lemma.

\section{Proofs of the theorems.}

Proof of Theorem 3. Let $F=f^{n}(z), G=f^{n}(q z)$. Then by Remark 2 we have, $S(r, f(q z))=$ $S_{1}(r, f)$. First suppose that $F \not \equiv G$. From Lemma 11 we get

$$
\begin{gathered}
\left(n p+n-1-\frac{n k+n+m+1}{m(n k+n-1)-1}\right)(\bar{N}(r, 0 ; F \mid \geq n p+n)+\bar{N}(r, 0 ; G \mid \geq n p+n)) \leq \\
\leq S(r, f)+S_{1}(r, f) .
\end{gathered}
$$

Noting that $n \geq 2$, from (10) we obtain

$$
\bar{N}(r, 0 ; F \mid \geq n p+n)+\bar{N}(r, 0 ; G \mid \geq n p+n) \leq S(r, f)+S_{1}(r, f),
$$

when $(2 p+1)\{m(2 k+1)-1\}>2 k+m+3$, i.e., when $2 m p k+(p+k)(m-1)>2$.

Similarly from Lemma 12 , when $2 m p k+(p+k)(m-1)>2$, we get

$$
\bar{N}(r, \infty ; F \mid \geq n k+n)+\bar{N}(r, \infty ; G \mid \geq n k+n) \leq S(r, f)+S_{1}(r, f) .
$$

Hence when $\psi_{p, k}^{m}>2$, adding (11) and (12) we see that only the second conclusion of Remark 4 holds. That is to say $f . g \equiv s$, where $0, \infty$ are lacunary values of $f$ and $g$ and $s^{n}=1$.

Suppose

$$
(f(z))^{n}(f(q z))^{n} \equiv 1
$$

From the fact that $f(z)$ and $f(q z)$ share $(\infty, k)$ and $(0, p)$, we conclude that $f(z)$ and $f(q z)$ both are entire functions and having no zeros.

So from (13) we have $N(r, 0 ; f) \leq S(r, f)$.

Therefore, in view of Lemmas 3 and 7 we have

$$
\begin{aligned}
2 n T(r, f) & =2 T(r, F)+O(1) \leq T\left(r, \frac{1}{F^{2}}\right)+S(r, f) \leq T\left(r, \frac{G}{F}\right)+S(r, f) \leq \\
& \leq T\left(r,\left(\frac{f(q z)}{f}\right)^{n}\right)+S(r, f) \leq n T\left(r, \frac{f(q z)}{f}\right) \leq S(r, f)+S_{1}(r, f),
\end{aligned}
$$

which is a contradiction. So we must have $F \equiv G$, that is $f^{n}(q z) \equiv f^{n}$. Then there exists a constant $t \in \mathbb{C}$ such that $f(z) \equiv t f(q z)$, where $t^{n}=1$.

Now we prove that $|q|=1$. Let $F(z)=f(z)^{n}$ and $F(q z)=f(q z)^{n}$, then we get $F(z)=$ $F(q z)$. Now replacing $z$ by $q z$, we get $F(q z)=F\left(q^{2} z\right)$ and so $F(z)=F\left(q^{2} z\right)$. Proceeding in the similar manner, we get $F(z)=F\left(q^{3} z\right)=F\left(q^{4} z\right)=\ldots=F\left(q^{m} z\right)$, where $m$ is an integer. Suppose that $|q|<1$. Then we get $F(z)=F\left(q^{m} z\right)$. Now for $m \longrightarrow \infty$, we see that $F(z)=F(0)$, which is a contradiction. We then suppose that $|q|>1$. Then after rewriting $F(z)=F(q z)$ by $F(c z)=F(z)$, where $c=\frac{1}{q}$, we also arrive at a contradiction. Hence, $|q|=1$ is the only possible conclusion. 
We omit the proofs of Theorems 1, 2 and 4 since they can be carried out in the line of proof of Theorem 3. For the proof of Theorem 1 and Theorem 4, it is necessary to use Lemmas 1 and 2 and in proof of Theorem 2 is is required to use Lemma 5 .

Acknowledgement. The authors wish to thank the referee for his/her valuable suggestions to wards the improvement of the paper. The research work of the first author is supported by DST-PURSE programme.

\section{REFERENCES}

1. A. Banerjee, S. Mukherjee, Weighted sharing of three sets, Southeast Asian Bull. Math., 34 (2010), 11-23.

2. D.C. Barnett, R.G. Halburd, R.J. Korhonen, W. Morgan, Nevanlinna theory for the q-difference operator and meromorphic solutions of q-difference equations, Proc. Roy. Soc. Edinburgh Sect., A, 137 (2007), no.3, 457-474.

3. Y.M. Chian, S.J. Feng, On the Nevanlinna Characteristic $f(z+\eta)$ and difference equations in complex plane, Ramanujan J., 16 (2008), 105-129.

4. F. Gross, C.F. Osgood, Entire functions with common preimages, in: Factorization theory of meromorphic functions and related topics, Lecture Notes in Pure and Appl. Math., Marcel Dekker, New York, 1982, 19-24.

5. R.G. Halburd, R.J. Korhonen, Difference analogue of the lemma on the logarithmic derivative with applications to difference equations, J. Math. Anal. Appl., 314 (2006), 477-487.

6. W.K. Hayman, Meromorphic Functions, The Clarendon Press, Oxford, 1964.

7. I. Lahiri, Value distribution of certain differential polynomials, Int. J. Math. Math. Sci., 28 (2001), no.2, 83-91.

8. I. Lahiri, Weighted sharing and uniqueness of meromorphic functions, Nagoya Math. J., 161 (2001), 193-206.

9. I. Lahiri, Weighted value sharing and uniqueness of meromorphic functions, Complex Var. Theory Appl., 46 (2001), 241-253.

10. X. Qi, L. Yang, Sets and value sharing of q-differences of meromorphic functions, Bull. Korean Math. Soc., 50 (2013), no.3, 731-745.

11. X. Qi, L. Yang, Sharing sets of q-difference of meromorphic functions, Math. Slovaca, 64 (2014), no.1, $51-60$.

12. J.F. Xu, X.B. Zhang, The zeros of q-difference polynomials of meromorphic functions, Adv. Diff. Equ., (2012) 2012:200, 1-10.

13. C.C. Yang, On deficiencies of differential polynomials, II, Math. Z., 125 (1972), 107-112.

14. H.X. Yi, Meromorphic functions that share three sets, Kodai Math. J., 20 (1997), 22-32.

15. H.X. Yi, C.C. Yang, Theory of the Uniqueness of Meromorphic Functions, Science Press, Beijing, 1995.

16. J.L. Zhang, Value distribution and shared sets of differences of meromorphic functions, J. Math. Anal. Appl., 367 (2010), no.2, 401-408.

17. J.L. Zhang, R. Korhonen, On the Nevanlinna characteristic of $f(q z)$ and its applications, J. Math. Anal. Appl., 369 (2010), 537-544.

Department of Mathematics, University of Kalyani

West Bengal, India

abanerjee_kal@yahoo.co.in, abanerjeekal@gmail.com

Department of Mathematics, University of Kalyani

West Bengal, India

saikat352@gmail.com, saikatbh89@yahoo.com 